\title{
Time Varying Betas And The Unconditional Distribution of Asset Returns
}

\author{
by \\ C J Adcock ${ }^{[1]}$, M Ceu Cortez ${ }^{[2]}$, M J Rocha Armada ${ }^{[2]}$ and Florinda Silva ${ }^{[2]}$ \\ [1] The University of Sheffield, UK; [2] The University of Minho, Braga, Portugal
}

This draft: January 2010

\begin{abstract}
This paper draws attention to the fact that under standard assumptions the time varying betas model cannot capture the dynamics in beta. Conversely, evidence of time variation in beta using this model is equivalent to non-normality in the unconditional distribution of asset returns. Using the multivariate normal as a model for the joint distribution of returns on market indices and predetermined information variables, it is shown how to capture skewness and kurtosis in the unconditional distributions of asset returns. Under the assumptions of the model, asset returns are unconditionally distributed as an extended quadratic form (EQF) in normal variables. Expressions are given for the moment generating function and for the computation of the distribution and density functions. The market-timing model is derived formally using this model. The properties of bias when the standard linear betas model is used to estimate alpha when the correct model is the EQF are also investigated. It is shown that a different time varying betas model can arise as a consequence of portfolio selection. It is also shown that the predetermined information variables have the potential to account for the time series properties of returns, including heterogeneity of variance. An empirical study applies the model to returns on $46 \mathrm{UK}$ bond funds. An analysis of the residuals shows that the model described in this paper is able to capture the dynamics of alpha and beta and properly account for other features of the time series of returns for 28 of these funds, of which 15 exhibit time variation in beta. The study reports the effect of the EQF model on the computation of VaR and CVaR and bias in the estimation of alpha.
\end{abstract}

Keywords: beta, elliptical symmetry, CVaR, kurtosis, market model, market timing, multivariate normality, quadratic forms in normal variables, skewness, VaR.

Address for correspondence

C J Adcock

University of Sheffield

9, Mappin Street

Sheffield, S1 4DT

UK

Tel: $\quad+44(0) 1142223402$

Fax: $\quad+44(0) 1142223348$

Email: c.j.adcock@shef.ac.uk 


\section{Introduction}

The aim of this paper is to present some new theoretical and empirical insights into a model that is widely used in financial economics for modelling the returns on risky financial assets. Specifically, the paper is concerned with extensions to the market model in which, in the usual notation, the parameters $\alpha$ and $\beta$ are time varying. In the case under consideration, the dynamics of $\alpha$ and $\beta$ are modelled by positing linear regression models in which the unobserved values of these two parameters are related to economic conditions by incorporating (what are generally referred to as) predetermined information variables. This model, which is described in detail in Section 2 of this paper, is both well known and widely used. Indeed, it may be regarded as a routine technique in asset pricing and portfolio theory as it is described in standard textbooks such as Elton et al. (2003, p. 150). The method itself is attributed to Beaver et al. (1970) and was popularised by Barr Rosenberg and his co-workers, for example Rosenberg and McKibben (1973) or Rosenberg and James (1976). The paper is motivated by the increasing application of this model to asset pricing studies (for example Jagannathan and Wang, 1996), specifically to mutual fund performance studies (for example Ferson and Schadt, 1996, Christopherson et al., 1998, Silva et al., 2003) and to the continuing interest in time variation in both alpha and beta (see Ferson et al., 2003, 2008)

The theoretical contribution of this paper is threefold. First, for the case when standard econometric assumptions hold, it is shown that the model is unable to describe the dynamics in $\beta$. Secondly, the paper describes the implications for modelling time varying $\alpha$ and $\beta$ when the standard econometric assumptions do not hold. This is done by proposing that the joint probability distribution of returns on market indices and the predetermined information variables (PIVs) is multivariate normal. Under these assumptions, the third theoretical contribution is to show that unconditionally returns are distributed as an extended quadratic form $(\mathrm{EQF})$ in normal variables. The use of quadratic forms is not widespread in financial economics, although there are some specific instances of their use. There are applications in option pricing theory, see Jaschke et al. (2002). A special case of a quadratic form, namely the difference of two independent Chi-squared variables, is well established in finance as the variance-gamma model of Madan and Seneta (1990) and Carr, Madan and Chang (1998). A quadratic form, which is related to time varying betas, arises as a model due to Dybvig and Ross (1985). This model describes portfolio returns in the situation where the manager has superior information and constructs the portfolio by maximising expected utility.

The first theoretical contribution therefore offers an explanation for those applications where the model has not provided evidence of time variation in $\alpha$ or $\beta$. More interestingly, for those applications, which have been successful, the two other contributions provide new insights into the unconditional distribution of asset returns and into the market model. In Section 3 it is shown that, under the model studied in this paper, the unconditional distribution of returns may include both skewness and kurtosis. It therefore offers new possibilities for portfolio selection as well as for modelling returns. One implication of non-normality in the unconditional distribution of returns is that the use of standard methods will lead to inaccurate computations of the percentiles and hence to inaccurate computations of standard measures of risk such as value at risk (VaR) and conditional value at risk $(\mathrm{CVaR})$. A second implication is the measurement of portfolio performance based on estimated values of alpha, the intercept term in the model. It is well known that 
errors in model specification can lead to misleading estimates of alpha. In this paper, the effect on the unconditional distribution of estimates of alpha is considered for the case when the correct model for returns is an extended quadratic form, but the model used for performance measurement is the ubiquitous linear beta model based on one or more market indices.

As noted above, quadratic forms in normal variables arise in the model of Dybvig and Ross (1985). In this model, standard assumptions are made about the distribution of asset returns and information variables. The quadratic form arises as a consequence of the portfolio selection process. In Section 4 of this paper, it is shown that a general version of Dybvig and Ross' model leads to an extended quadratic form for the unconditional distribution of portfolio returns. It also leads to time varying betas. However, in this case the temporal variation in beta is caused by the portfolio selection process and not by dependence on economic conditions through predetermined information variables.

The approach to time varying betas described in this paper leads to a version of the market model which includes a quadratic term in the return on the market portfolio. That is, it leads formally to the well-known market-timing model of Treynor and Mazuy (1966). The implication of this derivation of the model is that a manager will explicitly adjust beta to reflect the changes in the PIVs which cause its temporal variation. It is also shown that the time varying betas model offers the possibility of capturing both serial-correlation and heterogeneity in the variance of asset returns. A correctly specified time-varying betas model may therefore be able to account for the empirical properties that are often observed in the time series of returns on financial assets, even though the model is estimated using OLS or similar methods.

The model for the unconditional distribution of asset returns is illustrated using data on 45 individual UK bond funds and an equally weighted fund of funds, giving 46 portfolios in all. This data is from a larger study of bond fund performance evaluation reported in Silva et al. (2003). The structure of this paper is as follows. Section 2 describes the model under consideration and presents a short review of relevant literature. This section explains why the model fails under multivariate normality and related elliptically symmetric distributions. It also presents the new model and its basic properties. As noted above, this is based on the assumption that the joint probability distribution of returns on market indices and the information variables is multivariate normal. In section 3 , the EQF unconditional distribution of asset returns is presented, expressions are given for the moment generating function and for the computation of the distribution and density functions. Section 4 is concerned with bias in the estimation of alpha. Section 5 presents a time varying betas model based on the work of Dybvig and Ross (1985) in which the unconditional distribution of returns is also an EQF but where the temporal variation in beta arises as a consequence of portfolio selection. Section 6 shows that the well-known model of Treynor and Mazuy arises formally as the conditional distribution of returns given returns on the market index. Section 7 describes the empirical study. Section 8 concludes with a summary of the results and an indication of future work. There are four appendices with technical results that are used in Sections 2, 3, 4 and 6. Notation is that in standard use. In keeping with increasingly common practice, only the main results are presented. Further detail is available from the corresponding author on request. 


\section{The Model and its Properties}

The model for time varying betas has two components. The first is the market model in which returns on individual assets are related to returns on a proxy to the market portfolio. In the second component, linear models, in which the unobserved parameters are related to predetermined information variables (PIVs), represent the dynamics in the two parameters of the market model. The first component of the model is

$$
R_{t}=\alpha_{t}+\beta_{t} R_{m t}+\omega_{t}
$$

The notations $R_{t}$ and $R_{m t}$ are used indifferently to denote either total returns or excess returns on an asset and the market proxy respectively for the time period ending at time $t$. Excess return is defined in the usual way as total return minus the risk free return over the period. The time series of unobserved residual returns $\left\{\omega_{t}\right\}$ are assumed to be IID with zero mean and constant variance. In the remainder of the paper, the model defined by equation (1.) is referred to as the single index model.

The second component of the model describes the dynamics of $\alpha$ and $\beta$ using two linear models

$$
\alpha_{t}=\varphi_{0}+\varphi_{l}^{T} \mathbf{X}_{t-1}+\eta_{t}, \quad \beta_{t}=\gamma_{0}+\gamma_{l}^{T} \mathbf{X}_{t-1}+\varsigma_{t}
$$

In this pair of equations, $\mathbf{X}_{t-1}$ is a vector of (lagged) information variables (that is, it contains variables which are known at the start of period $t), \varphi_{1}$ and $\boldsymbol{\gamma}_{1}$ are the corresponding vectors of parameters, $\varphi_{0}$ and $\gamma_{0}$ are scalars. The residuals $\eta_{t}$ and $\zeta_{t}$ are unobserved. It is assumed that values of the 2 -vector $\left(\eta_{t}, \zeta_{t}\right)$ are IID and that it is distributed independently of $\omega_{t}$. Substitution of equation (2.) into equation (1.) gives the model

$$
R_{t}=\varphi_{0}+\varphi_{1}^{T} \mathbf{X}_{t-1}+\gamma_{0} R_{m t}+\gamma_{1}^{T} \mathbf{X}_{t-1} R_{m t}+\varepsilon_{t},
$$

where, by definition, the residual return $\varepsilon_{t}$ is given by

$$
\varepsilon_{t}=\omega_{t}+\eta_{t}+R_{m t} \varsigma_{\mathrm{t}}
$$

This model is used by Jagannathan and Wang (1996) in asset pricing and by Ferson and Schadt (1996) in a conditional performance evaluation framework. Both papers focus on the dynamics in beta. The dynamics of alpha are considered by Christopherson et al. (1998) and by Bernhardt and Jung (1979), who are concerned with statistical desirability of the inclusion of the intercept term. Ferson and Schadt (1996) further extend the model by incorporating a quadratic term in $R_{m t}$, thus obtaining a conditional version of the Treynor and Mazuy (1966) timing model

$$
R_{t}=\varphi_{0}+\boldsymbol{\varphi}_{1}^{T} \mathbf{X}_{t}+\gamma_{0} R_{m t}+\gamma_{1}^{T} \mathbf{X}_{t} R_{m t}+\gamma_{2} R_{m t}^{2}+\varepsilon_{\mathrm{t}},
$$


where $\gamma_{2}$ is a scalar. Ferson et al. (2008) are concerned with the effects of data mining and spurious regressions on the properties of alpha and beta. They report results which justify the use of models like that at equation (3.).

Given the specification above, the error terms $\left\{\varepsilon_{t}\right\}$ have zero mean but a variance that is time varying through their dependence on $R_{m t}$. Denoting the variance of the term $\left(\omega_{t}+\eta_{t}\right)$ by $\sigma_{\omega}^{2}$, the variance of $\varepsilon_{t}$ is

$$
V\left(\varepsilon_{t}\right)=\sigma_{\omega}^{2}+R_{m t}^{2} \sigma_{\varsigma}^{2}
$$

Generally, this complication is ignored and the models defined at equations (3.) or (4.) are estimated using OLS. A special case arises if all the elements of the parameter vector $\varphi_{1}$ are equal to zero. In this case, the alpha coefficient in equation (2.) is constant and only the beta is time varying. From the perspective of estimation, the non-linearity that arises because of the presence of the vector $\mathbf{X}_{t-1} R_{m t}$ may be ignored since, by specification, these terms are given. The model defined at equations (3.) and (4.) is applied to individual assets. If the vector $\mathbf{X}_{t-1}$ contains common factors, for example the change in interest rates, then it also applies to a portfolio of the assets. In this case the parameters $\varphi_{0}, \gamma_{0}, \varphi_{1}$, $\gamma_{1}$ and, if used, $\gamma_{2}$ will in general vary with each asset; giving parameters $\varphi_{0 i}$ and so on.

The single index model at (1.) may be replaced by a model in which there is more than one factor or explanatory variable. This may be motivated by the arbitrage pricing theory (Ross, 1976), or by empirical considerations. In such models, the scalar coefficient $\beta_{t}$ is replaced by a vector, $\boldsymbol{\beta}_{t}$. Similarly, the return on the market index $R_{m t}$ is replaced $\boldsymbol{R}_{m t}$ which denotes a vector of returns on several market indices. Such models are referred to as multi-index models. Time variation in the elements of $\boldsymbol{\beta}_{t}$ may be captured by positing vector linear models which are similar to the second component of equation of (2.)

$$
\boldsymbol{\beta}_{t}=\gamma_{0}+\Gamma_{l}^{T} \boldsymbol{X}_{t-1}+\varsigma_{t},
$$

where the vector $\varsigma_{t}$ has the multivariate normal distribution $\mathrm{N}\left(\boldsymbol{\theta}, \Sigma_{\varsigma}\right)$, which is distributed independently of $\eta_{t}$ and $\omega_{t}$. The corresponding model for $R_{t}$ is

$$
R_{t}=\varphi_{0}+\boldsymbol{\varphi}_{l}^{T} \mathbf{X}_{t}+\gamma_{0}^{T} \boldsymbol{R}_{m t}+\mathbf{X}_{t-1}^{T} \boldsymbol{\Gamma}_{l} \boldsymbol{R}_{m t}+\varepsilon_{t}
$$

where the residual return $\varepsilon_{t}$ and its variance are respectively

$$
\varepsilon_{t}=\omega_{t}+\eta_{t}+\boldsymbol{R}_{m t}^{T} \varsigma_{\mathrm{t}}, \sigma_{\varepsilon}^{2}=\sigma_{\omega}^{2}+\boldsymbol{R}_{m t}^{T} \Sigma_{\varsigma} \boldsymbol{R}_{m t} .
$$

Such models are referred to as CMI models, when alpha is constant, and ACMI models, when alpha is time varying. In what follows, unless stated otherwise, the developments that are described are presented in terms of the more general model at (5.) and (6.), which 
includes both (3.) and (4.) as special cases ${ }^{1}$. As already noted in section 2, it is normal practice to estimate the parameters of the model using OLS, that is ignoring the component of $\sigma_{\varepsilon}^{2}$ which depends on $\boldsymbol{R}_{m t}$. The justification for the use of OLS (or WLS) is that conditional on values of $\boldsymbol{R}_{m t}$ and $\boldsymbol{X}_{t-1}$ the probability distribution of $R_{t}$ is normal and that the expected value of the right hand side of equation (5.) exactly describes the conditional mean of $R_{t}$.

If it may be assumed that the variables $R_{t}, \boldsymbol{R}_{m t}$ and $\boldsymbol{X}_{t-1}$ have a joint multivariate normal distribution, then the models at equation (3.), (4.) and (5.) are all mis-specified. This is because the conditional distribution of $\mathrm{R}_{\mathrm{t}}$ given $\boldsymbol{R}_{m t}=\boldsymbol{r}_{m t}$ and $\boldsymbol{X}_{t-1}=\boldsymbol{x}_{t-1}$ has a mean that is strictly linear in $\boldsymbol{r}_{m t}$ and $\boldsymbol{x}_{t-1}$. This is a standard result in the theory of the multivariate normal distribution, which is summarised briefly in Appendix A. See, for example, Anderson (1958, p. 29) for further details. In the case of multivariate normality, using OLS to estimate the parameters of (5.) must give estimated values of the elements of the matrix $\boldsymbol{\Gamma}_{1}$ that are not significantly different from zero. If such a model is estimated and the null hypothesis $\mathrm{H}_{0}: \boldsymbol{\Gamma}_{1}=\boldsymbol{O}$ (where $\boldsymbol{O}$ is a matrix of zeros) is not rejected (against any suitable alternative), the implication is that the dynamics of $\boldsymbol{\beta}$, although not necessarily those of $\alpha$, must be captured another way ${ }^{2}$. The same comments apply to the quadratic model at (4.); the additional null hypothesis $\mathrm{H}_{0}: \gamma_{2}=0$ would not be rejected either.

These comments apply, with relatively minor modifications, if the joint multivariate probability distribution of $R_{t}, \boldsymbol{R}_{m t}$ and $\boldsymbol{X}_{t-1}$ comes from any member of the elliptically symmetric class. This includes the multivariate Student distribution, which is well established in finance (for example Chamberlain, 1983, Ingersoll, 1987, or Zhou, 1993) ${ }^{3}$, and the multivariate Laplace distribution (see Fang et al., 1990, p. 92). Under these distributions, the conditional mean of $R_{t}$ given $\boldsymbol{R}_{m t}=\boldsymbol{r}_{m t}$ and $\boldsymbol{X}_{t-1}=\boldsymbol{x}_{t-1}$ is unchanged and remains linear in $\boldsymbol{r}_{m t}$ and $\boldsymbol{x}_{t-1}$. The residual variance will in general be a function of the conditioning variables, with the precise form of the function depending on the joint distribution of these variables. Thus, it is possible to accommodate kurtosis in asset returns but to remain in the position where time variation in $\boldsymbol{\beta}$ cannot be captured by a model of the type defined at equations (3.), (4.) or (5.).

When the null hypothesis $\mathrm{H}_{0}: \boldsymbol{\Gamma}_{1}=\boldsymbol{O}$ is rejected (against any suitable alternative), the implication is that the unconditional distribution of $R_{t}$ will not be normal (more generally, it will not be from an elliptically symmetric distribution). Depending on the joint probability distribution of $\boldsymbol{R}_{m t}$ and $\boldsymbol{X}_{t-1}$, the unconditional distribution of $R_{t}$ will exhibit both skewness and kurtosis.

\footnotetext{
${ }^{1}$ For the sake of completeness, and following Ferson and Schadt (1996), the model at (5.) could include quadratic forms in $\boldsymbol{R}_{m t}$ and/or $\boldsymbol{X}_{t-1}$, thus formally extending the model at (4.). A possible motivation for this is quadratic behaviour in the underlying market model or in the models which relate alpha and beta to the PIVs.

${ }^{2}$ It may also be conjectured that failure to reject $\mathrm{H}_{0}$ is also a test of the joint multivariate normality of $R_{t}, \boldsymbol{R}_{m t}$ and $\boldsymbol{X}_{t-1}$, although this idea requires development which is beyond the scope of this paper.

${ }^{3}$ Further technical details of the results for the multivariate Student distribution are available on request.
} 
The conditional distribution of $R_{t}$ given $\boldsymbol{R}_{m t}=\boldsymbol{r}_{m t}$ and $\boldsymbol{X}_{t-1}=\boldsymbol{x}_{t-1}$ is normal. In the usual notation

$$
R_{t} \mid \boldsymbol{r}_{m t}, \boldsymbol{x}_{t-1} \sim N\left(\varphi_{0}+\boldsymbol{\varphi}_{1}^{T} \boldsymbol{x}_{t}+\boldsymbol{\gamma}_{0}^{T} \boldsymbol{r}_{m t}+\boldsymbol{x}_{t-1}^{T} \boldsymbol{\Gamma}_{l} \boldsymbol{r}_{m t}, \sigma_{\omega}^{2}+\boldsymbol{r}_{m t}^{T} \Sigma_{\zeta} \boldsymbol{r}_{m t}\right)
$$

In the rest of this paper it is assumed that the joint distribution of $\boldsymbol{R}_{m t}$ and $\boldsymbol{X}_{t-1}$ is multivariate normal. This approach, namely to make an exogenous assumption about the distribution of $\boldsymbol{R}_{m t}$ and $\boldsymbol{X}_{t-1}$ is similar to that adopted in Pedersen and Satchell (2000). To facilitate the presentation of the unconditional distribution of returns in Section 3 and the discussion of the model of Dybvig and Ross (1985) in Section 5, it is useful to define the vector $\boldsymbol{U}$ as

$$
\boldsymbol{U}=\left[\begin{array}{l}
\boldsymbol{R}_{m t} \\
\boldsymbol{X}_{t-1}
\end{array}\right] .
$$

The subscript $t$ is omitted. The mean vector and covariance matrix are denoted by $\boldsymbol{\mu}_{U}$ and $\Sigma_{U}$. These may be partitioned like $\boldsymbol{U}$ as

$$
\boldsymbol{\mu}_{U}=\left[\begin{array}{l}
\boldsymbol{\mu}_{m} \\
\boldsymbol{\mu}_{X}
\end{array}\right], \boldsymbol{\Sigma}_{U}=\left[\begin{array}{ll}
\boldsymbol{\Sigma}_{m m} & \boldsymbol{\Sigma}_{m X} \\
\boldsymbol{\Sigma}_{X m} & \boldsymbol{\Sigma}_{X X}
\end{array}\right] .
$$

\section{The Unconditional Distribution of Asset Returns}

The model at (7.) is written in terms of the vector $\boldsymbol{U}$ defined at (8.) as

$$
R=\varphi_{0}+\boldsymbol{\varphi}_{1}^{T} \boldsymbol{U}+\boldsymbol{U}^{T} \boldsymbol{\Phi}_{2} \boldsymbol{U}+\varepsilon
$$

where $\varepsilon$ is independently distributed as $N\left(0, \sigma_{\varepsilon}^{2}\right), \sigma_{\varepsilon}^{2}=\sigma_{\omega}^{2}+\boldsymbol{R}_{m t}^{T} \boldsymbol{\Sigma}_{\varsigma} \boldsymbol{R}_{m t}$. For the remainder of the paper, it is assumed that $\sigma_{\varepsilon}^{2}$ is constant. Henceforth, the vector $\boldsymbol{\varphi}_{1}$ is defined by gathering relevant terms from (7.). The matrix $\boldsymbol{\Phi}_{2}$ is defined as

$$
\boldsymbol{\Phi}_{2}=\frac{1}{2}\left[\begin{array}{cc}
0 & \boldsymbol{\Gamma}_{1} \\
\boldsymbol{\Gamma}_{1}^{T} & 0
\end{array}\right] .
$$

If they are required, other cross product and squared terms may be included in this notation by specifying non-zero matrices in the two diagonal blocks of $\boldsymbol{\Phi}_{2}$. Equation (9.) thus contains Ferson and Schadt's (1996) extension to include $R_{m t}^{2}$ as described at (4.). 
The random variable $Q=\boldsymbol{U}^{T} \boldsymbol{\Phi}_{2} \boldsymbol{U}$ is a quadratic form in normal variables. The probability distribution of $Q$ is complicated, but has been studied by many authors since the pioneering papers of Pearson et al. (1932) and Robbins and Pitman (1949). There is a comprehensive summary in the excellent monograph by Mathai and Prevost (1992) and introductory material in Johnson and Kotz (1970, Ch. 29). The moments and cumulants of the distribution of $Q$ may be recovered from the moment generating function, which is summarised below. A result by Gil-Pelaez (1951) concerning the inversion of Fourier transforms is used by Imhof (1961) to provide a method of computing both the distribution and density functions of Q. Imhof's method is simple to implement and, as Mathai and Prevost (1992, p. 142) point out, may be considered to be exact. The variable $R$ containing both linear and quadratic terms as defined above is an extended quadratic form (EQF henceforth) in normal variables. Adcock (2009) shows that Imhof's method may be extended to allow easy computation of both the distribution and density functions of $R$.

The rest of this section summarises the moment generating function of $R$, the general form of the distribution and the extension to Imhof's formula that is required to compute the density and distribution functions of $\mathrm{R}$.

\subsection{Moments and cumulants}

It is straightforward to show that the logarithm of the moment generating function or cumulant generating function of $R$ is

$$
\begin{aligned}
K_{R}(t)=\log E\left(e^{R t}\right)= & \varphi_{0} t+\sigma_{\varepsilon}^{2} t^{2} / 2+\boldsymbol{\mu}_{U}^{T} \boldsymbol{\Sigma}_{\boldsymbol{U}}^{-1}\left(\boldsymbol{\Sigma}_{\boldsymbol{U}}^{-1}-2 \boldsymbol{\Phi}_{2} t\right)^{-1} \boldsymbol{\Sigma}_{U}^{-1} \boldsymbol{\mu}_{U} / 2 \\
& +t^{2} \boldsymbol{\varphi}_{1}^{T}\left(\boldsymbol{\Sigma}_{\boldsymbol{U}}^{-1}-2 \boldsymbol{\Phi}_{2} t\right)^{-1} \boldsymbol{\varphi}_{1} / 2+t \boldsymbol{\mu}_{U}^{T} \boldsymbol{\Sigma}_{\boldsymbol{U}}^{-1}\left(\boldsymbol{\Sigma}_{U}^{-1}-2 \boldsymbol{\Phi}_{2} t\right)^{-1} \boldsymbol{\varphi}_{1} .
\end{aligned}
$$

In this notation, general expressions for the mean and variance of $R$ are, respectively

$$
E(R)=\boldsymbol{\varphi}_{1}^{T} \boldsymbol{\mu}_{U}+\operatorname{tr}\left(\boldsymbol{\Sigma}_{\mathbf{U}} \boldsymbol{\Phi}_{2}\right)+\boldsymbol{\mu}_{U}^{T} \boldsymbol{\Phi}_{2} \boldsymbol{\mu}_{U}
$$

and

$$
\operatorname{var}(R)=\sigma_{\varepsilon}^{2}+\varphi_{1}^{T} \boldsymbol{\Sigma}_{\mathbf{U}} \boldsymbol{\varphi}_{1}+2 \operatorname{tr}\left\{\left(\boldsymbol{\Sigma}_{\mathbf{U}} \Phi_{2}\right)^{2}\right\}+4 \mu_{U}^{T} \Phi_{2} \Sigma_{\mathbf{U}} \Phi_{2} \mu_{U}+2 \varphi_{1}^{T} \Sigma_{\mathbf{U}} \Phi_{2} \mu_{U}
$$

Higher moments may be recovered in the usual way.

\subsection{Exact Distribution of Returns}

The definition of the EQF in this paper is non-standard, because it includes both a linear function of $\boldsymbol{U}$ and a normal variable $\varepsilon$. It will also sometimes be the case that the matrix $\boldsymbol{\Phi}_{2}$ will be singular. It is shown in Appendix $C$ that $R$ may be written as 


$$
R=\varphi_{0}+\sum_{j=1}^{p} \lambda_{j} \chi_{\left(h_{j}\right)}^{2}\left(\theta_{j}\right)+\zeta
$$

where the $\chi_{\left(h_{j}\right)}^{2}\left(\theta_{j}\right)$ are independent non-central Chi-squared variables each with $h_{j}$ degrees of freedom and non-centrality parameters $\theta_{j}(\geq 0)$. The variable $\zeta$ is independently normally distributed as $N\left(0, \sigma_{\zeta}^{2}\right)$. The $\lambda_{j}$ are the $p$ distinct non-zero eigenvalues of the matrix $\boldsymbol{\Phi}_{2} \boldsymbol{\Sigma}_{U}$ and the $h_{j}$ their respective orders of multiplicity. The non-centrality parameters $\theta_{j}$ are functions of $\boldsymbol{\mu}_{\mathrm{U}}, \boldsymbol{\Sigma}_{\mathrm{U}}$ and the eigenvectors of the matrix $\mathbf{H}^{\mathrm{T}} \boldsymbol{\Phi}_{2} \mathbf{H}$, where $\mathbf{H}$ is a non-singular matrix satisfying $\mathbf{H H}^{\mathrm{T}}=\boldsymbol{\Sigma}_{\mathrm{U}}$, the so-called square root matrix.

\subsection{Computing the Distribution and Density Function}

The distribution function of $R, F_{R}($.$) in the usual notation, may be computed by direct$ inversion of the characteristic function. For the standard quadratic form

$$
\sum_{j=1}^{p} \lambda_{j} \chi_{\left(h_{j}\right)}^{2}\left(\theta_{j}\right)
$$

Gil-Pelaez's (1951) representation of the inversion formula for Fourier transforms is used by Imhof (1961) to provide a simple expression for the distribution function. The expression is in terms of a real integral which may be computed to an arbitrary degree of accuracy using a suitable numerical procedure such as the trapezoidal rule. A similar integral, derived by differentiation under the integral sign, leads to the corresponding result for the density function of $R$. There are many procedures reported in the literature for the computation of the distribution of quadratic forms. As noted above Imhof's method is easy to implement and, as Mathai and Prevost (1992, page 142) point out, it may be considered to be exact. For the extended quadratic form at (12.), Adcock (2009) provides an extension to Imhof's procedure. The integral for the distribution function of $R$ is

$$
F(r)=\frac{1}{2}-\frac{1}{\pi} \int_{0}^{\infty} \frac{\sin \delta(s) \mathrm{e}^{-\sigma_{\zeta}^{2} \mathrm{~s}^{2} / 8}}{\mathrm{~s} \rho(\mathrm{s})} d s,
$$

where

$$
\begin{aligned}
& \delta(s)=\frac{1}{2} \sum_{j=1}^{p}\left\{h_{j} \tan ^{-1}\left(\lambda_{j} s\right)+\theta_{j} \lambda_{j} s\left(1+\lambda_{j}^{2} s^{2}\right)^{-1}\right\}-\frac{1}{2} r s, \\
& \rho(s)=\prod_{j=1}^{p}\left(1+\lambda_{j}^{2} s^{2}\right)^{h_{j} / 4} \exp \left\{\frac{1}{2} \sum_{j=1}^{p} \theta_{j} \lambda_{j}^{2} s^{2} /\left(1+\lambda_{j}^{2} s^{2}\right)\right\} .
\end{aligned}
$$

The corresponding expression for the density function is 


$$
f(r)=\frac{1}{2 \pi} \int_{0}^{\infty} \frac{\cos \delta(s) \mathrm{e}^{-\sigma_{s^{2}}^{2} / 8}}{\rho(s)} d s .
$$

\section{Bias in the Estimation of Alpha}

As noted in the introduction, the linear beta model is ubiquitous. That is, the model

$$
R_{t}=\alpha+\boldsymbol{\beta}^{T} \boldsymbol{R}_{m t}+\omega_{t}
$$

is widely used to estimate the alpha of a portfolio. If the correct model for returns is as specified at equation (5.) or, in a different notation, equation (9.), omission of the PIVs and the cross products of PIVs with the market indices will cause bias in the estimator of $\alpha$ if (13.) is used. This section of the paper gives two methods for testing the extent of the bias. The first is based on the unconditional distribution of returns; that is the EQF at (9.). The second method uses the fact that the model at (5.) is linear in its parameters. Standard regression methods may therefore be used to estimate and test the bias.

Assuming for simplicity that the estimator of $\boldsymbol{\beta}$ is replaced by its true value and in the usual notation, the estimator of $\alpha$ is

$$
\hat{\alpha}=\bar{R}-\boldsymbol{\beta}^{T} \overline{\boldsymbol{R}}_{m}
$$

If the linear beta model is correctly specified, $\hat{\alpha}$ is unconditionally distributed at $N\left(\alpha, \sigma_{\omega}^{2} / T\right)$, where $T$ is the sample size. Using the notation at equation (8.), this may be written as

$$
\hat{\alpha}=\bar{R}-\tilde{\boldsymbol{\beta}}^{T} \overline{\boldsymbol{U}}, \tilde{\boldsymbol{\beta}}^{T}=\left(\boldsymbol{\beta}^{T}, \boldsymbol{O}^{T}\right),
$$

where $\boldsymbol{0}$ denotes a vector of zeros. Using (9.) this may be written as

$$
\hat{\alpha}=\varphi_{0}+\left(\boldsymbol{\varphi}_{1}-\tilde{\boldsymbol{\beta}}\right)^{T} \overline{\boldsymbol{U}}+T^{-1}\left(\sum_{t=1}^{T} \boldsymbol{U}_{t}^{T} \boldsymbol{\Phi}_{2} \boldsymbol{U}_{t}\right)+\bar{\varepsilon} .
$$

That is, under the extended quadratic form model, $\hat{\alpha}$ is unconditionally distributed as the mean $\bar{Q}$ of the IID extended quadratic forms

$$
Q_{t}=\varphi_{0}+\left(\boldsymbol{\varphi}_{1}-\tilde{\boldsymbol{\beta}}\right)^{T} \boldsymbol{U}_{t}+\boldsymbol{U}_{t}^{T} \boldsymbol{\Phi}_{2} \boldsymbol{U}_{t}+\varepsilon_{t}
$$

The assumption about $\boldsymbol{\beta}$ made above implies that the sample size $T$ is large enough for the central limit theorem to ensure that $\bar{Q}$ has a normal distribution. In this case, if $S_{Q}^{2}$ denotes the sample variance of the $Q_{t}$, the significance of $\alpha$ may be tested by the statistic $Z=\sqrt{T} \bar{Q} / S_{Q}$. 
The second method assumes that $\boldsymbol{\beta}$ in the model at (13.) is estimated by $\hat{\boldsymbol{\beta}}$ using OLS in the usual way. This method is used in the empirical study. The estimator of $\alpha$ is

$$
\hat{\alpha}=\bar{R}-\hat{\boldsymbol{\beta}}^{T} \overline{\boldsymbol{R}}_{m}
$$

The properties of this estimator, which may be obtained by standard regression manipulations, are summarised in Appendix D using standard regression notation. It is shown that conditional on the omitted independent variables in $\boldsymbol{U}$, bias in $\hat{\alpha}$ is normally distributed. Appendix D gives the mean and variance of the distribution of $\hat{\alpha}$ when, as above, it is assumed that the slope parameters in the regression are given. The variance for the case when it is necessary to take into account estimation error in the regression is also given.

\section{Portfolio Selection and Extended Quadratic Forms}

Dybvig and Ross (1985), henceforth D\&R, consider portfolio selection for the case where there is one risky and one risk free asset with return $r_{f}$. The model for the return on the risky asset is

$$
R=r_{f}+\pi+S+\varepsilon
$$

where $\pi$ denotes the risk premium. The random variables $S$ and $\varepsilon$ are independently normally distributed with zero means and variances $\sigma_{S}^{2}$ and $\sigma_{\varepsilon}^{2}$ respectively. The manager has access to superior information and observes the signal $S$. If the manager maximises expected utility using an exponential utility function with risk aversion coefficient $\varphi$, D\&R show that portfolio return may be written as

$$
R_{p}=\left(\tilde{\pi}^{2}+2 \tilde{\pi} S+\tilde{\pi} \varepsilon+S \varepsilon+S^{2}\right) / \varphi \sigma_{\varepsilon}^{2}, \tilde{\pi}=\pi+r_{f} .
$$

This is an extended quadratic form in the two variables $S$ and $\varepsilon$. Interestingly, D\&R correctly make the point that $S^{2}$ is distributed proportionally to a Chi-squared variable (with one degree of freedom) and thus induces skewness in returns. However, they do not note that the cross product term $S \mathcal{\varepsilon}$ may have the effect of removing skewness when it appears in conjunction with $S^{2}$. This section presents a more general formulation of the $\mathrm{D} \& \mathrm{R}$ model. The aim of the section is to demonstrate that portfolio returns follow an EQF distribution when the returns on individual assets are normally distributed and that there is a time varying alphas and betas representation.

Let $\mathbf{R}$ be a vector of returns on a universe of assets. The time subscript is omitted. A model for $\mathbf{R}$ is the multivariate linear regression

$$
\mathbf{R}=\boldsymbol{\delta}+\mathbf{B U}+\varepsilon, \varepsilon \sim N\left(\mathbf{0}, \Sigma_{\varepsilon}\right)
$$

where $\mathbf{U}$ is the vector of variables defined at (8.). The usual regression assumptions are made, the expected value of $\mathbf{R}$ is given by $\boldsymbol{\delta}+\mathbf{B U}$ and the covariance matrix, which is 
assumed to be non-singular, equals $\boldsymbol{\Sigma}_{\varepsilon}$. Setting appropriate columns of $\mathbf{B}$ to zero means that the model above may include or exclude information variables and one or more market indices. When information variables are included, this model implies that alpha is time varying. The betas, however, are constant. On applying Stein's lemma (Stein, 1981) and maximising expected utility subject to the budget constraint, the vector of portfolio weights is given by

$$
w=w_{0}+\theta \Pi B U
$$

where $1 / \theta$ represents risk aversion,

$$
\boldsymbol{w}_{0}=\left(\boldsymbol{\Sigma}_{\varepsilon}^{-1} \mathbf{l} / \mathbf{1}^{T} \boldsymbol{\Sigma}_{\varepsilon}^{-1} \mathbf{l}\right)+\theta \boldsymbol{\Pi} \boldsymbol{\delta}, \boldsymbol{\Pi}=\left\{\boldsymbol{\Sigma}_{\varepsilon}^{-1}-\left(\boldsymbol{\Sigma}_{\varepsilon}^{-1} \mathbf{1} \mathbf{1}^{T} \boldsymbol{\Sigma}_{\varepsilon}^{-1} / \mathbf{1}^{T} \boldsymbol{\Sigma}_{\varepsilon}^{-1} \mathbf{l}\right)\right\},
$$

and $\boldsymbol{I}$ is a vector of ones. This is a standard result, which dates from Merton (1972), but is presented in a notation which is suitable for what follows. Portfolio return is $R_{p}=\mathbf{R}^{T} \boldsymbol{w}$. This is an extended quadratic form in the vector of variables formed by $\mathbf{U}$ and $\boldsymbol{\varepsilon}$ and is given by

$$
R_{p}=(\boldsymbol{\delta}+\mathbf{B U}+\boldsymbol{\varepsilon})^{T}\left(\boldsymbol{w}_{\boldsymbol{0}}+\theta \mathbf{\Pi B U}\right)
$$

The case where $\mathbf{R}=\boldsymbol{\delta}+\boldsymbol{\varepsilon}, \boldsymbol{\varepsilon} \sim \boldsymbol{N}\left(\boldsymbol{0}, \boldsymbol{\Sigma}_{\varepsilon}\right)$ is considered in Adcock (2000). For the general case, there is more than one way of decomposing portfolio return, but straightforward manipulations give a time varying betas model

$$
R_{p}=\alpha+\boldsymbol{\beta}^{T} \mathbf{U}+\eta
$$

where

$$
\alpha=\boldsymbol{\delta}^{T}\left(\boldsymbol{w}_{0}+\theta \mathbf{\Pi B U}\right), \boldsymbol{\beta}=\mathbf{B}^{T}\left\{\boldsymbol{w}_{0}+\theta \boldsymbol{\Pi}(\mathbf{B U}+\boldsymbol{\varepsilon})\right\}, \eta=\boldsymbol{w}_{0}^{T} \boldsymbol{\varepsilon}
$$

This model exhibits time variation in both the portfolio alpha and betas. The time variation arises as a consequence of the portfolio selection process and the models for alpha and the vector of betas are formulated using the same variables, $\mathbf{U}$, used in the underlying model for asset returns. The vector of unobserved residuals, $\boldsymbol{\varepsilon}$, appears in the model for $\boldsymbol{\beta}$ as well as in the model for asset returns.

Detailed consideration of this model is beyond the scope of this paper, although some brief comments are warranted. First, it may be considered to be more restricted than the time varying betas model at equation (5.) which is the main subject of this work. This is because the specification above implies that the parameters in the linear relationship between returns and the variables in the vector $\mathbf{U}$ do not exhibit temporal variation. Secondly, the normality assumption and Stein's lemma mean that the results above, as well as those in D\&R, hold for any well-behaved utility function ${ }^{4}$. Furthermore, the

\footnotetext{
${ }^{4}$ The utility function $U(R)$ should be differentiable twice and both $E\left(U^{\prime}\right)$ and $E\left(U^{\prime}\right)$ must exist.
} 
extension of Stein's lemma due to Landsman and Nešlehová 2008) means that they also hold in principle if the vector of residuals $\varepsilon$ comes from an elliptically symmetric distribution with covariance matrix proportional to $\Sigma_{\varepsilon}$. Finally, some refinement of this model would be required for practical purposes. This is because the portfolio selection process uses a vector of variables which also forms part of the subsequent return computation. In practice, portfolio selection at the start of period $t$ will always use information available at the end of period $t-1$. This condition is satisfied by the PIVs in $\mathbf{U}$, but not by the returns on the market indices which are contemporaneous with $\mathbf{R}$ the vector of asset returns.

\section{The Conditional Distribution of Asset Returns and the Market Model}

The development described in this section is concerned with extensions of the market model, that is the conditional distribution of $R_{t}$ given $\boldsymbol{R}_{m t}$ only. The conditional distribution of $R_{t}$ given $\boldsymbol{X}_{t-1}$ is also briefly discussed as this model has the capability to describe time series properties that are often observed in asset returns.

The conditional distribution of $R_{t}$ given $\boldsymbol{R}_{m t}=\boldsymbol{r}_{m t}$ and $\boldsymbol{X}_{t-1}=\boldsymbol{x}_{t-1}$ is given by equation (7.). Since the conditional variance does not depend on $\boldsymbol{x}_{t-1}$, the conditional distribution of the return on an asset $R_{t}$ given market return $\boldsymbol{r}_{m t}$ is also normal. It is shown in appendix B that conditional expected value and conditional variance are both quadratic functions of $\boldsymbol{r}_{m t}-\boldsymbol{\mu}_{m}$, namely

$$
E\left(R_{t} \mid \boldsymbol{r}_{m t}\right)=\psi_{0}+\boldsymbol{\psi}_{1}^{T} \tilde{\boldsymbol{r}}_{m t}+\tilde{\boldsymbol{r}}_{m t}^{T} \boldsymbol{\Psi}_{2} \tilde{\boldsymbol{r}}_{m t}, \operatorname{var}\left(R_{t} \mid \boldsymbol{r}_{m t}\right)=\lambda_{0}+2 \lambda_{1}^{T} \tilde{\boldsymbol{r}}_{m t}+\tilde{\boldsymbol{r}}_{m t}^{T} \boldsymbol{\Lambda}_{2} \tilde{\boldsymbol{r}}
$$

where $\tilde{\boldsymbol{r}}_{m t}=\boldsymbol{r}_{m t}-\boldsymbol{\mu}_{m}$. The scalars $\psi_{0}, \lambda_{0}$, vectors $\boldsymbol{\psi}_{1}, \lambda_{1}$ and matrices $\boldsymbol{\Psi}_{2}$ and $\boldsymbol{\Lambda}_{2}$ are defined in Appendix B. In the case of a single market index, when $\boldsymbol{R}_{m t}$ is a scalar, the conditional expected value in equation (14.) is the market-timing model of Treynor and Mazuy (1966) or the model proposed in Harvey and Siddique (2000) to capture skewness. For this case, the implication of (14.) is that a portfolio manager who correctly adjusts the beta of a portfolio to reflect changes caused by the PIVs will exhibit a positive timing coefficient.

Using exactly the same approach, it is straightforward to derive the conditional expected value and variance of $R_{t}$ given that $\boldsymbol{X}_{t-1}=\boldsymbol{x}_{t-1}$. The equations are omitted in the interests of brevity. Recalling that, by specification, the elements of $\boldsymbol{X}_{t-1}$ are known at the start of the time period, this representation is able to account for heterogeneity in both the conditional mean and variance of returns and thus has the capability of playing a role similar to that of a member of the ARCH family of models. Depending on the properties of $\boldsymbol{X}_{t-1}$, it also offers the possibility of accounting for serial correlation.

\section{Empirical Study}


The model for time varying betas and the ensuing results for the unconditional distribution of asset returns were originally motivated by the increasing application of models with time-varying alphas and betas to fund performance evaluation. The implication of the results reported in Section 2 is that a correctly specified model will result in estimated residuals that satisfy the standard diagnostic tests used in time series regression. The main result reported in Section 3 at equation (12.) shows that the unconditional distribution of asset returns will be determined by the non-zero eigenvalues of the matrix $\boldsymbol{\Phi}_{2} \boldsymbol{\Sigma}_{U}$, the corresponding non-centrality parameters and $\sigma_{\zeta}^{2}$, the variance of the zero-mean normally distributed variable $\zeta$. There are cases, as shown below, where the parameters in equation (12.) result in an unconditional distribution which is almost normal, particularly in its tails. In general, however, the unconditional distribution of returns may exhibit fattails or skewness or both. The implication of non-normality in the unconditional distributions is that the use of standard methods will lead to inaccurate computations of the percentiles and hence to inaccurate computations of standard measures of risk such as value at risk $(\mathrm{VaR})$ and conditional value at risk $(\mathrm{CVaR})$.

In this paper, the results reported in Sections 2 and 3 and in particular the effects on percentiles of the unconditional distribution of returns are illustrated using data on $46 \mathrm{UK}$ bond portfolios. The sample of $45 \mathrm{UK}$ funds consists mainly of Gilt funds and corporate bond funds. A small number of "Other Bond" funds is also included and an equally weighted fund of funds. This data is from a larger study of bond fund performance evaluation reported in Silva et al. (2003), which also covers 5 other European markets and almost 600 other bond funds. UK bond funds have been used in this paper because it was shown in Silva et al.'s paper to be one of the markets for which funds presented more evidence of time-variation in the betas. As is shown below, the unconditional distribution of returns for some bond funds exhibit different and interesting characteristics. The model we use for this purpose is the ACMI as described in equation (5.). Three market indices and 2 PIVs are included in this model. All tests have been carried out at the $95 \%$ level of significance.

This section contains a short description of the data, including the predetermined information variables, and summarises the model estimation and testing procedures. The results of the study are then presented. These cover the results of model estimation and subsequent computations based on the unconditional distribution of returns. The section includes three graphs which display the unconditional return density function for three funds.

Monthly data for UK bond funds investing mainly in the domestic market over the period February 1994 to December 2000 was obtained from Datastream. All fund returns are monthly continuously compounded returns, with income distributions reinvested. In order to obtain excess returns, the risk free rate, proxied by 3-month LIBOR, is subtracted from this return. The excess return on the Salomon Smith Barney WGBI all maturities for the UK market (UKBindex), the excess return on the UK MSCI stock index (UKSindex) ${ }^{5}$ and a default spread (calculated as the difference between the MSCI Euro Credit Index BBB

\footnotetext{
${ }^{5}$ The excess return on the stock index is included as it can be viewed as a measure of expectations about general economic conditions (see Elton et al, 1995, and Cornell and Green, 1991) and also because some of the funds can hold a small percentage of stocks.

$-13-$
} 
rated and the MSCI Euro Credit Index AAA rated) ${ }^{6}$ are considered as market indices. The PIVs used as conditioning information were the term spread and inverse relative wealth $(I R W)$. Previous research has motivated the choice of these variables (Ilmanen, 1995, Silva et al., 2003). The term spread is the difference between the yield on a 10 -year UK Government bond and the 3-month LIBOR. IRW is the ratio between the exponentially weighted average of past real wealth and current wealth. The MSCI UK stock price index deflated by the Consumer Price Index (obtained from the International Monetary Fund) is used to measure real wealth. These two variables (term spread and IRW) are stochastically de-trended (by subtracting a 12-month moving average) ${ }^{7}$ and mean zero variables. Table 1 presents main descriptive statistics for the bond funds.

\section{Table 1 - Summary statistics on fund returns - about here}

According to the Bera-Jarque test statistic, the hypothesis of a normal distribution is rejected (at the 5\% level) for 62 percent of the funds. The equally weighted portfolio of funds also exhibits evidence of non-normality.

The parameters of the ACMI model described at equation (5.) were estimated using OLS. Estimates of the corresponding parameters of the extended quadratic form representation at (9.) were computed by direct substitution. The eigenvalues and related parameters required for the canonical representation at (12.) were also computed. After fitting the ACMI model, the estimated residuals were tested for normality, GARCH effects and for serial correlation using a battery of standard tests. Of the 46 UK bond funds, 28 have estimated residuals which satisfy the assumptions of the model at (5.). The estimated residuals of the remaining 19 funds exhibited a combination of GARCH effects, kurtosis or skewness. Since this has implications both for parameter estimation and for the unconditional distribution of asset returns, these 19 funds are excluded from further consideration in the present paper. Extension of the model to accommodate these effects is a topic for future work. For the 28 funds, the significance of the parameters in (5.) which correspond to the non-linear terms in the model was tested using the F-to-enter test. The results of the tests for the 28 funds are shown in Table 2. The table is divided into two panels. Panel (i) reports results for 15 funds for which the F-to-enter test indicates that there are significant non-linear coefficients in the models; that is, there is statistically significant evidence of time variation in beta. Panel (ii) reports results for 13 funds for which there is no such evidence. It is interesting to note that the equally weighted fund of funds exhibits time variation in its betas and its estimated residuals satisfy the diagnostic tests. Since by specification it includes 19 funds whose residuals do not satisfy these tests, this is an example of the statistical benefits of diversification and the central limit theorem.

\section{Table 2 - Diagnostic tests for funds with estimated IID normal residuals - about here}

This table reports the probability of the F-to-enter test, the Bera-Jarque test and a likelihood ratio test for $\operatorname{GARCH}(1,1)$ effects. For the Bera-Jarque test, the skewness and

\footnotetext{
${ }^{6}$ This spread for the aggregate Euro zone is used as a measure of the default risk that may affect corporate bond returns.

7 This procedure was used in order to reduce the problem of spurious regression, a problem that may be found when persistent regressors are used (see Ferson et al., 2003a, 2003b).
} 
kurtosis components and their p-values are reported separately as well as the overall test statistic and p-value. As a robustness check, the significance of the non-linear coefficients in (5.) was also examined using the Wald test. As this gave very similar results to the F-toenter test the details are omitted, but are available on request from the corresponding author. Tests were also performed for serial correlations, but no evidence of this was found in any of the 46 funds $^{8}$. In should be noted that Table 2 and the succeeding tables are based on the 28 funds which satisfy the diagnostic tests. There is therefore an element of selection bias, which means that results should be interpreted with a degree of caution. Of course, the same caveat applies to the 18 funds which did not pass the diagnostic tests.

Table 3 gives a comparison of percentiles based on the fitted EQF model at (12.) with an assumed normal distribution. For a range of nominal probabilities from $0.1 \%$ to $99.9 \%$, the table shows the absolute difference in probability as a percentage. This was computed as follows. For each fund, the sample mean and variance were used to compute the percentiles for each of the ten probabilities shown using an assumed normal distribution. The probability under the fitted EQF model was then computed using the method described in Section 3. The table is divided into two panels in the same way as Table 2.

\section{Table 3 - Absolute error in the percentiles of the unconditional distribution of asset returns - about here}

The entries in Table 3 may appear deceptively small. Closer examination reveals that the average absolute difference in panel (i) is about $80 \%$ higher than in panel (ii). This suggests that the non-linear terms, equivalently evidence of time variation in beta, do indeed have an effect on the percentiles of the return distribution. A different view may be obtained by presenting the results as a percentage of the nominal tail probability. This is shown in Table 4.

\section{Table 4 - Absolute error in the percentiles of the unconditional distribution of asset returns as percentages - about here}

Not surprisingly the percentage errors in the extreme tails are substantial. Of more practical consequence are the errors in the $1 \%$ and $5 \%$ columns since these are the nominal probability levels commonly used for $\mathrm{VaR}$ and $\mathrm{CVaR}$ computations. In panel (i), for example, the UK fund of funds (UKallfunds) exhibits relatively small percentage errors, except at the extremes. This suggests that the unconditional distribution of its returns is well approximated by the normal distribution at least in the region of the $1 \%$ and $5 \%$ points, even though there is significant evidence of time variation in beta. For fund $U K b f 32$, the percentage errors are substantial at all the tabulated probability levels suggesting non-normality in the unconditional distribution. Graphs of the return distributions are shown below for these two funds.

Table 5 shows the comparison of percentiles re-expressed as differences in $\mathrm{VaR}$ and CVaR. The table is divided into two panels as above. The first two columns of the table give the 1-percentile of the distribution based on the estimated EQF and an assumed normal distribution, respectively. The difference in the $1 \% \mathrm{VaR}$ based on a fund of 100 million monetary units is shown in column three. The absolute percentage difference

\footnotetext{
${ }^{8}$ Similarly, there is no evidence of serial correlation in the three market indices.
} 
compared to the VaR based on assumed normal returns is shown in column 4. The monetary difference in $\mathrm{VaR}$ at $5 \%$ is shown in column 5 and the two corresponding CVaRs in columns six and seven.

\section{Table 5 - Differences in VaR and CVaR at $1 \%$ and $5 \%$ - about here}

The differences in both $1 \%$ columns are generally negative, thus showing that the normal assumption would understate both $\mathrm{VaR}$ and $\mathrm{CVaR}$. At $5 \%$ the picture is more mixed; there are several positive differences. This illustrates the ability of the EQF model to detected peakedness around the mean and implies that the use of the normal distribution could cause an overstatement of $\mathrm{VaR}$ and/or $\mathrm{CVaR}$ at $5 \%$ for some funds. Interpretation of the significance of the monetary differences in $\mathrm{VaR}$ and $\mathrm{CVaR}$ is not a main purpose of this paper. The maximum absolute difference shown in the table is just over 950,000 units, less than $1 \%$ of the assumed fund value. Whether this would be regarded as a serious monetary difference is a matter for the fund manager and the clients. However, the results shown in column 4 indicate that the percentage differences can be substantial. For $U K b f 32$ the difference is over 650,000 units or $12 \%$ of the $\mathrm{VaR}$ computed using the normal assumption. For UKallfunds the percentage difference is much smaller. Graphs of the probability density functions of UKbf32 and UKallfunds based on the estimated EQF model are shown in Figures 1 and 2.

\section{Figures 1 and 2 - about here}

Figure 1 shows the departure from normality and a small degree of asymmetry is visible. Figure 2 shows that the distribution of returns for the fund of funds is close to normality except in the centre of the distribution. Similar graphs for other funds are available on request.

The results in panels (ii) of Tables 3, 4 and 5 are for funds for which, according to the results of the F-to-enter test in Table 2, there are no significant non-linear effects. Under the assumptions of the model the unconditional distribution of returns is normal. Theoretically, for funds in panels (ii) the $\mathrm{VaR}$ and $\mathrm{CVaR}$ computations based on the EQF model may be taken to be statistically equivalent to those based on the assumed normal model. Indeed, panels (ii) of Tables 3, 4 and 5 generally show that the differences from the normal model are small. However, there are exceptions. For example, UKbf40 exhibits a substantial difference in $\mathrm{VaR}$ and $\mathrm{CVaR}$ at the $1 \%$ level. A graph of the density function for $U K b f 40$ is shown in Figure 3. A degree of asymmetry is clearly seen, even though this is not indicated by the tests reported in Table 2 .

\section{Figure 3 - about here}

The implication of this is that the EQF model can result in an unconditional distribution which exhibits non-normality even though the test of the non-linear coefficients in the model at (5.) fail to reveal a departure from normality. Resolution of this inconsistency is a topic for future work.

Table 6 - Comparison of probabilities in the left hand tail - about here 
Table 6 summarises the differences in probabilities for these three funds at four levels of probability in the left hand tail of the EQF distribution. The table shows the probabilities computed for a normal distribution with the same mean and variance based on the exact quantiles from the EQF distribution.

\section{Table 7 - Bias in the estimation of alpha - about here}

Table 7 presents an analysis of the bias caused by using a standard linear market model at equation (13.) based on the three market indices. Column 1 of the table shows estimated values of alpha based on (13.). The second column shows the p-value of the standard $\mathrm{t}$ test. Columns 3 and 4 show the corresponding data based on the ACMI model at (5.). Columns 5 through 7 show the bias in alpha, its standard error computed using equation (B) of Appendix D and the resulting test statistic. The table is divided into four panels. The first two panels contain the 15 funds for which there is evidence of time variation in beta and which satisfy the diagnostic tests. The third and fourth panels contain the 13 funds which satisfy the diagnostic tests but for which there is no evidence of time variation in the betas. Panels (i) and (iii) show funds with statistically significant values of alpha estimated using (13.). Panels (ii) and (iv) show funds for which the estimated values of alpha are not significantly different from zero. Within each panel, funds are presented in increasing order of the p-value in column 2. It may be noted that, correct to four decimal places, all estimated values of alpha are less than or equal to zero.

According to the $\mathrm{Z}$ test column of Table 7, there is no evidence that use of the model at (13.) causes significant bias in alpha. This finding holds even for the 7 funds in panel (i) for which there is statistically significant evidence of time variation in beta and for which the linear model at (13.) results in statistically significant values of alpha. There is one other point to note, which is that use of the distribution at (C) of Appendix D would further reduce the magnitude of the $\mathrm{Z}$ test statistics for this sample of funds.

\section{Conclusions}

Using the multivariate normal as a model for the joint distribution of returns on market indices and the predetermined information variables, it is shown how to capture skewness and kurtosis in the unconditional distributions of asset returns. Under the assumptions of the model, asset returns are unconditionally distributed as an extended quadratic form (EQF) in normal variables. Formulae are given for the moment generating function and first two moments. It is shown how to compute both the distribution and density functions by numerical inversion of the characteristic function.

The paper investigates the properties of bias when the standard linear betas model is used to estimate alpha when the correct model is in fact the EQF. The paper also presents a generalisation of a model due to Dybvig and Ross and shows how to formulate it as a time-varying betas model. In this case, time variation in betas is due to the portfolio selection process and not dependence on economic factors. Further investigation of this model is a topic for future work. The well-known Treynor and Mazuy market-timing model arises as the conditional distribution of returns given the return on the market indices. It is also shown that the predetermined information variables have the potential to account for the time series properties of returns, including heterogeneity of variance. 
The empirical study applies the model to returns on 46 UK bond funds. An analysis of the residuals shows that the model described in this paper is able to capture the dynamics of alpha and beta and properly account for other features of the time series of returns for 28 of these funds, of which 15 exhibit time variation in beta. The study reports the effect of the EQF model on the computation of $\mathrm{VaR}$ and $\mathrm{CVaR}$. An investigation into estimates of alpha which arise from the use of a standard linear market model suggests that the resulting bias is not statistically significant.

For the remaining 18 funds, analysis of the residuals suggests the presence of GARCH effects or non-normality or both. Future developments of these models will investigate these problems. Non-normality per se suggests that the use of non-normal distributions for the residuals may be required. The use of GARCH models is standard in conjunction with regression but leaves unresolved the question of computing the unconditional distribution of returns. As noted in Section 7, there are some inconsistencies in the results of the significance testing and the subsequent computations of percentiles of the distribution. Another further planned development is the correct treatment of the residual variances, which according to the model have a temporal dependence on the returns on the market indices.

\section{Acknowledgements}

The authors are grateful to the referees of the previous version of this paper. Their comments and suggestions have led both to improvements in the text and the technical content of the work.

\section{Appendices}

\section{A - The multivariate normal distribution}

Consider an $n+p+1$ vector $\mathbf{Y}$ which is partitioned as

$$
\mathbf{Y}=\left[\begin{array}{c}
\mathbf{R} \\
\tilde{\mathbf{X}}
\end{array}\right],
$$

where $\mathbf{R}$ is an $n$ vector which denotes the return on all assets and $\tilde{\mathbf{X}}$ is a vector of length $p+1$ defined as

$$
\tilde{\mathbf{X}}=\left[\begin{array}{c}
\mathbf{R}_{\mathrm{m}} \\
\mathbf{X}
\end{array}\right]
$$

The time subscript is omitted. The vector of expected values and the covariance matrix are similarly partitioned as

$$
\boldsymbol{\mu}_{\mathrm{Y}}=\left[\begin{array}{l}
\boldsymbol{\mu}_{\mathrm{R}} \\
\boldsymbol{\mu}_{\tilde{\mathrm{X}}}
\end{array}\right], \quad \boldsymbol{\Sigma}=\left[\begin{array}{ll}
\boldsymbol{\Sigma}_{\mathrm{RR}} & \boldsymbol{\Sigma}_{\mathrm{R} \tilde{\mathrm{X}}} \\
\boldsymbol{\Sigma}_{\mathrm{R} \tilde{\mathrm{X}}}^{\mathrm{T}} & \boldsymbol{\Sigma}_{\tilde{\mathrm{X}} \tilde{\mathrm{X}}}
\end{array}\right] .
$$


It is assumed that $\boldsymbol{\Sigma}, \boldsymbol{\Sigma}_{\mathrm{RR}}$ and $\boldsymbol{\Sigma}_{\tilde{\mathrm{X}} \tilde{\mathrm{X}}}$ are all of full rank. If the vector $\mathbf{Y}$ has a multivariate normal distribution, the conditional distribution of $\mathbf{R}$ given that $\tilde{\mathbf{X}}=\tilde{\boldsymbol{x}}$ is also multivariate normal with expected value

$$
\boldsymbol{\mu}_{\mathrm{R} \mid \tilde{\mathrm{X}}}=\boldsymbol{\mu}_{\mathrm{R}}+\boldsymbol{\Sigma}_{\mathrm{R} \tilde{\mathrm{X}}} \boldsymbol{\Sigma}_{\tilde{\mathrm{X}} \tilde{\mathrm{X}}}^{-1}\left(\tilde{x}-\boldsymbol{\mu}_{\tilde{\mathrm{X}}}\right)=\boldsymbol{\mu}_{\mathrm{R}}+\mathbf{B}\left(\tilde{\boldsymbol{x}}-\boldsymbol{\mu}_{\tilde{\mathrm{X}}}\right)
$$

and covariance matrix

$$
\boldsymbol{\Sigma}_{\mathrm{R} \mid \tilde{\mathrm{X}}}=\boldsymbol{\Sigma}_{\mathrm{RR}}-\boldsymbol{\Sigma}_{\mathrm{R} \tilde{\mathrm{X}}} \boldsymbol{\Sigma}_{\tilde{\mathrm{X}} \tilde{\mathrm{X}}}^{-1} \boldsymbol{\Sigma}_{\mathrm{R} \tilde{\mathrm{X}}}^{\mathrm{T}}=\boldsymbol{\Sigma}_{\mathrm{RR}}-\mathbf{B} \boldsymbol{\Sigma}_{\tilde{\mathrm{X}} \tilde{\mathrm{X}}} \mathbf{B}^{\mathrm{T}}
$$

For further details, see Anderson (1958, p. 29).

\section{B - Conditional distribution of returns given returns on the market indices}

On applying the results in appendix A and using the notation of Section 2, the conditional mean vector and covariance matrix of $\boldsymbol{X}_{t-1}$ given that $\boldsymbol{R}_{m t}=\boldsymbol{r}_{m t}$ are

$$
\begin{gathered}
\boldsymbol{\mu}_{X \mid r_{m}}=\boldsymbol{\mu}_{X}+\boldsymbol{B}_{m}\left(\boldsymbol{r}_{m t}-\boldsymbol{\mu}_{m}\right)=\boldsymbol{\mu}_{X}+\boldsymbol{B}_{m} \tilde{\boldsymbol{r}}_{m t}, \\
\Sigma_{X \mid r_{m}}=\Sigma_{X X}-\Sigma_{X m} \Sigma_{m m}^{-1} \Sigma_{m X},
\end{gathered}
$$

where $\boldsymbol{B}_{m}=\Sigma_{X m} \Sigma_{m m}^{-1}$. The conditional expected value of $R_{t}$ given $\boldsymbol{r}_{m t}$ is

$$
\mathrm{E}\left(R_{t} \mid \boldsymbol{r}_{m t}\right)=\varphi_{0}+\boldsymbol{\gamma}_{0}^{T} \boldsymbol{r}_{m t}+\left(\boldsymbol{\varphi}_{1}^{\mathrm{T}}+\boldsymbol{r}_{m t}^{T} \boldsymbol{\Gamma}_{1}^{T}\right) \boldsymbol{\mu}_{X \mid r_{m}}
$$

This may be written as

$$
E\left(R_{t} \mid \mathbf{r}_{m t}\right)=\psi_{0}+\boldsymbol{\psi}_{1}^{T} \tilde{\mathbf{r}}_{m t}+\tilde{\mathbf{r}}_{m t}^{T} \boldsymbol{\Psi}_{2} \tilde{\mathbf{r}}_{m t},
$$

where the constants are defined as

$$
\begin{gathered}
\psi_{0}=\varphi_{0}+\boldsymbol{\gamma}_{0}^{T} \boldsymbol{\mu}_{\boldsymbol{m}}+\left(\boldsymbol{\varphi}_{1}^{T}+\boldsymbol{\mu}_{m}^{T} \boldsymbol{\Gamma}_{l}^{T}\right) \boldsymbol{\mu}_{X,} \\
\psi_{l}=\boldsymbol{\gamma}_{0}+\boldsymbol{\Gamma}_{l}^{T} \boldsymbol{\mu}_{X}+\left(\boldsymbol{\varphi}_{1}^{T}+\boldsymbol{\mu}_{m}^{T} \boldsymbol{\Gamma}_{l}^{T}\right) \boldsymbol{B}_{m,} \\
\boldsymbol{\Psi}_{2}=\boldsymbol{\Gamma}_{l}^{T} \boldsymbol{B}_{m} .
\end{gathered}
$$

The conditional variance is

$$
V\left(R_{t} \mid \boldsymbol{r}_{m t}\right)=\sigma_{\omega}^{2}+\boldsymbol{r}_{m t}^{T} \Sigma_{\varsigma} \boldsymbol{r}_{m t}+\left(\boldsymbol{\varphi}_{1}^{T}+\boldsymbol{r}_{m t}^{T} \boldsymbol{\Gamma}_{l}^{T}\right)^{T} \Sigma_{X \mid r_{m}}\left(\boldsymbol{\varphi}_{1}+\boldsymbol{\Gamma}_{l} \boldsymbol{r}_{m t}\right)
$$

This may be written as 


$$
V\left(R_{t} \mid r_{m t}\right)=\lambda_{0}+2 \lambda_{l}^{T} \tilde{\boldsymbol{r}}_{m t}+\tilde{\boldsymbol{r}}_{m t}^{T} \boldsymbol{\Lambda}_{2} \tilde{\boldsymbol{r}}_{m t}
$$

where

$$
\begin{gathered}
\lambda_{0}=\sigma_{\omega}^{2}+\left(\boldsymbol{\varphi}_{1}+\boldsymbol{\Gamma}_{l} \boldsymbol{\mu}_{m}\right)^{T} \Sigma_{X \mid r_{m}}\left(\boldsymbol{\varphi}_{1}+\boldsymbol{\Gamma}_{l} \boldsymbol{\mu}_{m}\right) \\
\lambda_{1}^{T}=\left(\boldsymbol{\varphi}_{1}+\boldsymbol{\Gamma}_{l} \boldsymbol{\mu}_{m}\right)^{T} \Sigma_{X \mid r_{m}} \boldsymbol{\Gamma}_{l}, \\
\boldsymbol{\Lambda}_{2}=\Sigma_{\varsigma}+\boldsymbol{\Gamma}_{l}^{T} \Sigma_{X||_{m}} \boldsymbol{\Gamma}_{l .}
\end{gathered}
$$

\section{C - Distribution of quadratic forms and related results}

This appendix lists a number of results which are required in Section 3. The results are standard, but are presented in the notation of the paper. A general reference is Mathai and Prevost (1992) who summarise many of the well-known and well-established results in this area. It is assumed that the covariance matrix $\boldsymbol{\Sigma}$ is non-singular; the minor modifications that are required for the singular case are omitted.

\section{Theorem 1}

Let $\boldsymbol{U}$ be an $n$ vector with the full rank multivariate normal distribution $N(\boldsymbol{\mu}, \boldsymbol{\Sigma})$ and let $Q$ be the quadratic form $Q=\boldsymbol{U}^{T} \boldsymbol{\Phi} \boldsymbol{U}$ where without loss of generality the matrix $\boldsymbol{\Phi}$ is symmetric. $Q$ Is distributed as

$$
\sum_{j=1}^{p} \lambda_{j} \chi_{\left(h_{j}\right)}^{2}\left(\theta_{j}\right)
$$

where the $\chi_{\left(h_{j}\right)}^{2}\left(\theta_{j}\right)$ are independent non-central Chi-squared variables each with $h_{j}$ degrees of freedom and non-centrality parameters $\theta_{j}(\geq 0)$. The $\lambda_{j}$ are the $p$ distinct nonzero eigenvalues of the matrix $\mathbf{\Phi} \boldsymbol{\Sigma}$ and the $h_{j}$ their respective orders of multiplicity. The non-centrality parameters $\theta_{j}$ are

$$
\theta_{j}=\boldsymbol{\mu}^{T} \mathbf{H}^{T} \boldsymbol{t}_{j} \boldsymbol{t}_{j}^{T} \mathbf{H} \boldsymbol{\mu}
$$

where $\boldsymbol{t}_{j}$ is the eigenvector of $\mathbf{H}^{\mathrm{T}} \mathbf{\Phi H}$ corresponding to $\lambda_{j}$ and where $\mathbf{H}$ is a nonsingular matrix satisfying $\mathbf{H H}^{\mathrm{T}}=\boldsymbol{\Sigma}$.

Let $\boldsymbol{U}$ and $\boldsymbol{\Phi}$ be as defined for Theorem 1 and let $\tilde{Q}$ be the extended quadratic form (EQF) $\tilde{Q}=\varphi^{\mathrm{T}} \mathbf{U}+\mathbf{U}^{T} \mathbf{\Phi} \mathbf{U}$. 


\section{Corollary 1}

If $\boldsymbol{\Phi}$ is non-singular the variable $\tilde{\mathrm{Q}}+\boldsymbol{\varphi}^{\mathrm{T}} \boldsymbol{\Phi}^{-1} \boldsymbol{\varphi} / 4$ is distributed as the quadratic form $\tilde{\mathbf{U}}^{T} \boldsymbol{\Phi} \tilde{\mathbf{U}}$ where $\tilde{\mathbf{U}}$ has the multivariate normal distribution $N(\tilde{\boldsymbol{\mu}}, \boldsymbol{\Sigma})$ with $\tilde{\boldsymbol{\mu}}=\boldsymbol{\mu}+\boldsymbol{\Phi}^{-1} \boldsymbol{\varphi}_{1} / 2$.

\section{Theorem 2}

If $\boldsymbol{\Phi}$ is singular, $\tilde{Q}$ is distributed as $\boldsymbol{d}^{\mathrm{T}} \mathbf{W}+\mathbf{W}^{\mathrm{T}} \boldsymbol{\Lambda} \mathbf{W}$ where $\mathbf{W}$ is distributed as $N(\boldsymbol{\omega}, \mathbf{I}), \boldsymbol{\omega}=\mathbf{T}^{\mathrm{T}} \mathbf{H}^{-1} \boldsymbol{\mu}, \boldsymbol{\Lambda}$ is the diagonal matrix of the eigenvalues $\lambda_{j}$ of $\mathbf{H}^{\mathrm{T}} \boldsymbol{\Phi} \mathbf{H}, \mathbf{T}$ is the matrix of the corresponding eigenvectors $\boldsymbol{t}_{j}, \boldsymbol{d}=\mathbf{T}^{\mathrm{T}} \mathbf{H}^{\mathrm{T}} \boldsymbol{\varphi}$ and $\mathbf{I}$ is a unit matrix.

\section{Corollary 2}

Let $A$ denote the values of $j$ for which the eigenvalues are non-zero and let $\bar{A}$ denote its complement. The EQF may be written as

$$
Z=\sum_{j \in A} \lambda_{j}\left(W_{j}+d_{j} / 2 \lambda_{j}\right)^{2}+\sum_{j \in A} d_{j} W_{j}-\sum_{j \in A} d_{j}^{2} / 4 \lambda_{j}
$$

with empty sums being interpreted as zero. The first term is a weighted sum of non-central Chi-squared variables, the non-centrality parameters being

$$
\left\{\boldsymbol{t}_{j}^{\mathrm{T}}\left(\mathbf{H}^{-1} \boldsymbol{\mu}+\mathbf{H}^{\mathrm{T}} \boldsymbol{\varphi} / 2 \lambda_{j}\right)\right\}^{2}
$$

The second term is an independently distributed normal variable whose mean and variance are, respectively, $\sum_{j \in \bar{A}} d_{j} \omega_{j}$ and $\sum_{j \in \bar{A}} d_{j}^{2}$.

\section{D - Bias arising from omitted variables}

In this appendix, standard regression notation is used. Conditional on the market indices and predetermined information variables, the model at (5.) is linear in its parameters and may be written as

$$
\boldsymbol{y}=\alpha \boldsymbol{I}+\boldsymbol{V} \boldsymbol{\beta}+\boldsymbol{W} \boldsymbol{\delta}+\boldsymbol{\varepsilon}, \boldsymbol{\varepsilon} \sim N\left(\boldsymbol{0}, \sigma^{2} \mathbf{I}\right)
$$

where $\boldsymbol{y}$ denotes the vector of returns on the asset or fund, $\boldsymbol{V}$ denotes the matrix of observations on the market indices, $\boldsymbol{W}$ denotes the matrix of observations on the PIVs and the cross products of PIVs with the indices and I is a $T \times T$ unit matrix. Let $\tilde{\boldsymbol{V}}$ and $\tilde{\boldsymbol{W}}$ denote the two matrices when the variables are corrected for the respective sample means $\overline{\boldsymbol{v}}$ and $\overline{\boldsymbol{w}}$ and let $\boldsymbol{I}$ denote a vector of ones. 
When only the market indices are included in the model, the estimator of alpha is

$$
\hat{\alpha}=\bar{y}-\overline{\boldsymbol{v}}^{T} \hat{\boldsymbol{\beta}}, \hat{\boldsymbol{\beta}}=\left(\tilde{\boldsymbol{V}}^{T} \tilde{\boldsymbol{V}}\right)^{-1} \tilde{\boldsymbol{V}}^{T} \boldsymbol{y}
$$

This may be written in terms of the model at (A). Rearrangement gives

$$
\hat{\alpha}=\alpha+T^{-1} \boldsymbol{1}^{T} \mathbf{M}(\boldsymbol{W} \boldsymbol{\delta}+\boldsymbol{\varepsilon}), \mathbf{M}=\left\{\boldsymbol{I}-\boldsymbol{V}\left(\tilde{\boldsymbol{V}}^{T} \tilde{\boldsymbol{V}}\right)^{-1} \tilde{\boldsymbol{V}}^{T}\right\} .
$$

Conditional on $\boldsymbol{\delta}$ and the values of $\boldsymbol{V}$ and $\boldsymbol{W}, b(\hat{\alpha})$, the bias in $\hat{\alpha}$, is normally distributed as

$$
N\left(T^{-1} \boldsymbol{1}^{T} \mathbf{M W} \boldsymbol{\delta}, T^{-2} \boldsymbol{\sigma}^{2} \boldsymbol{1}^{T} \mathbf{M M}^{\mathbf{T}} \boldsymbol{1}\right) .
$$

The bias is consistently estimated by $\hat{b}=T^{-1} \boldsymbol{I}^{T} \mathbf{M W} \hat{\boldsymbol{\delta}}$, where $\hat{\boldsymbol{\delta}}$ is the OLS estimator of $\boldsymbol{\delta}$ from (A). Straightforward manipulations show that the variance of the distribution of $\hat{b}$ which takes into account the effect of estimating $\boldsymbol{\delta}$, is

$$
\operatorname{var}(\hat{b})=T^{-2} \boldsymbol{\sigma}^{2} \boldsymbol{1}^{T}\left(\mathbf{I}+\mathbf{W} \mathbf{K}^{-1} \tilde{\mathbf{W}}^{T}\right) \mathbf{M} \mathbf{M}^{T}\left(\mathbf{I}+\mathbf{W} \mathbf{K}^{-1} \tilde{\mathbf{W}}^{T}\right)^{T} \boldsymbol{1}
$$

where $\mathbf{K}=\tilde{\boldsymbol{W}}^{T} \tilde{\boldsymbol{W}}-\tilde{\boldsymbol{W}}^{T} \tilde{\boldsymbol{V}}\left(\tilde{\boldsymbol{V}}^{T} \tilde{\boldsymbol{V}}\right)^{-1} \tilde{\boldsymbol{V}}^{T} \tilde{\boldsymbol{W}}$. In both cases $\sigma^{2}$ is estimated by the residual variance of the regression at (A).

\section{References}

Adcock, C. J. (2000) Dynamic Control of Risk in Optimised Portfolios. The IMA Journal of Mathematics Applied in Business and Industry, 11, p127-138.

Adcock, C. J. (2009) Computing the Distribution of an Extended Quadratic Form in Normal Variables Using Imhof's Method, Working Paper.

Anderson, T. W.(1958) Introduction to Multivariate Statistical Analysis, New York, John Wiley \& Sons Inc.

Bernhardt, I. and B. S. Jung (1979) The Interpretation of Least Squares Regression With Interaction or Polynomial Terms, The Review of Economics and Statistics, 61, p481-483.

Beaver, W., P. Kettler and M. Scholes (1970) The Association Between Market Determined and Accounting Determined Risk Measures, The Accounting Review, 45, p654-682.

Carr, P.P., D. B. Madan and E. C. Chang (1998). The Variance Gamma Process and Option Pricing, European Finance Review, 2, p79-105.

Chamberlain, G. (1983) A Characterisation of the Distributions that Imply Mean-Variance Utility Functions, Journal of Economic Theory, 29, p185-201.

Christopherson, J. A., W. E. Ferson and D. A. Glassman (1998) Conditioning Managers Alphas on Economic Information: Another Look at the Persistence of Performance, The Review of Financial Studies, 11, p111-142.

Cornell, B. and K. Green (1991) The Investment Performance of Low-Grade Bond Funds, The Journal of Finance, 46, p29-48. 
Dybvig, P. H. and S. A. Ross (1985) Differential Information and Performance Measurement Using a Security Market Line, The Journal of Finance, 40, p383399.

Elton, E. J., M. J. Gruber, S. J. Brown and W. N. Goetzmann (2003) Modern Portfolio Theory and Investment Analysis, Sixth Edition, New York, John Wiley \& Sons Inc.

Elton, E. J.; M. J. Gruber and C. R. Blake (1995) Fundamental Economic Variables, Expected Returns, and Bond Fund Performance, The Journal of Finance, 50, p1229-1256.

Fang K.-T., S. Kotz and K.-W. Ng (1990) Symmetric Multivariate and Related Distributions, London, Chapman and Hall.

Ferson, W. E. and R. W. Schadt (1996) Measuring Fund Strategy and Performance in Changing Economic Conditions, The Journal of Finance, 51, p425-461.

Ferson, W. E., S. Sarkissian and T. Simin (2003), Spurious Regressions in Financial Economics?, The Journal of Finance, 58, p1393-1413.

Ferson, W. E., S. Sarkissian and T. Simin (2008)Asset Pricing Models with Conditional Betas and Alphas: The Effects of Data Snooping and Spurious Regression, The Journal of Financial and Quantitative Analysis, 43, p331-354.

Gil-Pelaez, J. (1951) Note on the inversion theorem, Biometrika, 38, p481-482.

Ilmanen, A. (1995) Time-varying Expected Returns in International Bond Markets, The Journal of Finance, 50, p481-506.

Imhof, J. P. (1961) Computing the Distribution of Quadratic Forms in Normal Variables, Biometrika, 48, p419-426.

Ingersoll, J. E. (1987) Theory of Financial Decision Making, Savage, Maryland, Rowman and Littlefield.

Jagannathan, R. and Z. Wang (1996) The Conditional CAPM and the Cross-section of Expected Returns, The Journal of Finance, 51, p3-53.

Jaschke, S., C. Klüppelberg and A. Lindner (2002) Asymptotic Behavior of Tails and Quantiles of Quadratic Forms of Gaussian Vectors, Working Paper.

Johnson, N. R. and S. Kotz (1970) Distributions in Statistics, Continuous Univariate Distributions - 2, Boston, Houghton-Mifflin.

Harvey, C. R. and A. Siddique (2000) Conditional Skewness in Asset Pricing Tests, Journal of Finance, 55, p1263-1295.

Landsman, Z. \& J. Nešlehová (2008) Stein's Lemma for elliptical random vectors, Journal of Multivariate Analysis, 99, p912 - 927.

Madan, D.B. and E. Seneta (1990). The variance gamma (V.G.) model for share market returns, The Journal of Business, 63, p511-524.

Mathai, A.M. and S. B. Prevost (1992) Quadratic Forms in Random Variables, Heidelberg, Springer.

Merton, R. (1972). An Analytical Derivation of the Efficient Portfolio Frontier, Journal of Financial and Quantitative Analysis, 7, p1851-1872.

Pearson, K., S. A. Stouffer and F. N. David (1932) Further Applications in Statistics of the $\mathrm{T}_{\mathrm{m}}(\mathrm{x})$ Bessel Function, Biometrika, 24, p293.350.

Pedersen, C. S. and S. E. Satchell (2000) Small Sample Analysis of Performance Measures in the Asymmetric Response Model, Journal of Financial and Quantitative Analysis, 35, p425-450.

Robbins, H. and E. J. G. Pitman (1949) Application of the Method of Mixtures to Quadratic Forms in Normal Variates, Annals of Mathematical Statistics, 20, p552-560. 
Rosenberg, B. and W. McKibben (1973) The Prediction of Systematic and Specific Risk in Common Stocks, Journal of Financial and Quantitative Analysis, 8, p317-333.

Rosenberg, B. and G. James (1976) Prediction of Beta From Investment Fundamentals, Financial Analysts Journal, 32, p60-72.

Ross, S. A.(1976) The Arbitrage Theory of Capital Asset Pricing, Journal of Economic Theory, 13, p341-360.

Silva, F. C., M. C. Cortez and M. R. Armada (2003) Conditional Information and European Bond Fund Performance, European Financial Management, 9, p201230.

Stein, C. (1981) Estimation of the Mean of a Multivariate Normal Distribution, Annals of Statistics, 9, p1135-1151.

Treynor, J. L. and K. Mazuy (1966) Can Mutual Funds Outguess the Market? Harvard Business Review, 44, p131-136.

Zhou G. (1993) Asset-Pricing Tests Under Alternative Distributions, Journal of Finance, 48, p1927-1942. 